\title{
Physiological Properties of Newly Formed Synapses Between Sympathetic Preganglionic Neurons and Sympathetic Ganglion Neurons
}

\author{
Richard I. Hume* and Marcia G. Honigt \\ Department of Biology, University of Michigan, Ann Arbor, Michigan 48109
}

\section{SUMMARY}

We have examined the physiological properties of transmission at newly formed synapses between sympathetic preganglionic neurons and sympathetic ganglion neurons in vitro. Chick neurons were labeled with fluorescent carbocyanine dyes before they were placed into culture (Honig and Hume, 1986), and were studied by making intracellular recordings during the first 2 weeks of coculture. Evoked monosynaptic excitatory postsynaptic potentials (EPSPs) were not observed until $48 \mathrm{~h}$ of coculture. Beyond this time, the frequency with which connected pairs could be found did not vary greatly with time.

With repetitive stimulation, the evoked monosynaptic EPSPs fluctuated in amplitude from trial to trial and showed depression at frequencies as low as $1 \mathrm{~Hz}$. To gain further information about the quantitative properties of transmission at newly formed synapses, we analyzed the pattern of fluctuations of delayed release EPSPs. In mature systems, delayed release EPSPs are known to repre- sent responses to single quanta, or to the synchronous release of a small number of quanta. For more than half of the connections we studied, the histograms of delayed release EPSPs were extremely broad. This result suggested that either quantal reponses are drawn from a continuous distribution that has a large coefficient of variation or that there are several distinct size classes of quantal responses. The pattern of fluctuations of monosynaptic EPSPs was consistent with both of these possibilities, and was inconsistent with the possibility that monosynaptic EPSPs are composed of quantal subunits with very little intrinsic variation. Although variation in the size of responses to single quanta might arise in a number of ways, one attractive explanation for our results is that the density and type of acetylcholine receptors varies among the different synaptic sites on the surface of developing sympathetic ganglion neurons.

\section{INTRODUCTION}

The only synapse for which a detailed timetable of synaptic development is available is the skeletal neuromuscular junction. At this cholinergic synapse, both the pre- and postsynaptic cells are highly differentiated before they make contact. Thus

Received July 16, 1990; accepted November 26, 1990

Journal of Neurobiology, Vol. 22, No. 3, pp. 249-262 (1991)

(C) 1991 John Wiley \& Sons, Inc.

CCC 0022-3034/91/030249-14\$04.00

* To whom correspondence should be addressed. Include Natural Science Building with above address.

† Present address: Department of Anatomy and Neurobiology, University of Tennessee, Memphis, Memphis, Tennessee 38163 . muscle cells can express acetylcholine receptors before neurons arrive (Sytkowski, Vogel, and Nirenberg, 1973; Bevan and Steinbach, 1977), and presynaptic neurons can package and release transmitter before they encounter a muscle cell (Hume, Role, and Fischbach, 1983; Young and Poo, 1983). A consequence of this precocious development is that functional interactions can be seen as soon as the growth cone arrives at the muscle surface (Frank and Fischbach, 1979; Kidokoro and Yeh, 1982; Sun and Poo, 1987). However, even neuromuscular synapses undergo an extended period of maturation during which their functional properties are extensively modified (see Purves and Lichtman, 1985 for a review). We wondered whether the stages of synaptogenesis are similar in 
other developing systems. The properties of cholinergic synapses between preganglionic axons and autonomic neurons have been extensively studied in mature animals, and there is strong evidence that the basic mechanisms of synaptic transmission at these neuronal synapses are quite similar to those described for the neuromuscular junction (Blackman, Ginsborg, and Ray 1963a,b; Martin and Pilar, 1964; Dennis, Harris, and Kuffler, 1971; Bornstein 1974; 1978; McLachlan, 1975; Dreyer and Chiappinelli, 1987). To begin to address whether the process of synaptogenesis is also similar, we have examined the functional properties of synapses developing in vitro between sympathetic preganglionic neurons and their normal target neurons from sympathetic ganglia.

\section{MATERIALS AND METHODS}

\section{Culture Methods}

All experiments were performed on chick neurons in dissociated cell culture. The cells of interest were labeled with long-lasting fluorescent carbocyanine dyes. The details of the labeling procedure have already been described (Honig and Hume, 1986). Briefly, preganglionic neurons were retrogradely labeled in stage $30-32 \mathrm{em}-$ bryos with the red fluorescent dye, Dil ( 1,1 '-dioctadecyl$3,3,3^{\prime}, 3^{\prime}$-tetramethylindocarbocyanine perchlorate) and sympathetic ganglion neurons from stage $35-36 \mathrm{em}$ bryos were labeled by incubation in a dilute solution of the green fluorescent dye, DiO (3,3'-dioctadecyloxacarbocyanine perchlorate). Sympathetic ganglion neurons were labeled either at the time of plating, or 1 day prior to addition of preganglionic neurons. By viewing the cultures with epifluorescence illumination through the appropriate filters, we could positively identify neurons as belonging to one of three classes of cells: preganglionic neurons, sympathetic ganglion neurons, and all other spinal cord cells.

Our culture methods were similar to those we have previously described (Honig and Hume, 1986) with the following exceptions: (1) collagenase, rather than trypsin, was used to dissociate the cells of the spinal cord, (2) in most experiments a substrate of laminin atop polyornithine (Moorman and Hume, 1990) was used, and (3) the medium used to feed cultures contained $2 \%$ heat-inactivated horse serum but no embryo extract or muscleconditioned medium. Medial spinal cord alone was used to make cultures, so motor neurons were not present. Suspensions of spinal cord cells containing labeled preganglionic neurons (from stage 30-32 embryos) were added to cultures of sympathetic ganglion neurons ( from stage 35-36 embryos) that had been established 1-20 days previously. Typically, 50,000 spinal cord cells (containing about 1000-2000 labeled preganglionic neurons) were added to cultures containing 10,000 ganglion cells per dish. Thus, there were usually several ganglion cells in each field of view that contained a preganglionic neuron.

We made recordings from preganglionic neurons and sympathetic ganglion neurons using conventional intracellular techniques. Micropipets were usually filled with $300 \mathrm{~m} \mathrm{M} \mathrm{KCl}$, but we sometimes used electrodes with $5 \%$ Lucifer Yellow ( $\mathrm{Li}$ salt) or $5 \%$ horseradish peroxidase (HRP) (in $300 \mathrm{~m} \mathrm{M} \mathrm{KCl}$ ) so that we could determine the morphology of the cells under study. HRP-filled cells were processed by the Hanker Yates technique (Hanker, Yates, Metz, and Rustioni, 1977). We usually viewed our cultures with a $40 \times$ phase contrast objective, for which the diameter of the field of view was $450 \mu \mathrm{m}$. The axons of preganglionic neurons in culture can extend for over $1 \mathrm{~mm}$ (Honig and Hume, 1986), so all ganglion neurons in the same field of view as a preganglionic neuron were potential target cells.

All recordings were made at room temperature. The solution that bathed the cells contained (in $\mathrm{m} M$ ) 132 , $\mathrm{NaCl} ; 1.3, \mathrm{NaH}_{2} \mathrm{PO}_{4} ; 5.3, \mathrm{KCl} ; 1.7, \mathrm{MgSO}_{4} ; 5.4, \mathrm{CaCl}_{2}$; 12 , HEPES; 6.3 , glucose; and 0.3 , phenol red. The recording solution had an elevated calcium concentration ( 5.4 $\mathrm{m} M$ compared to normal level of about $2 \mathrm{~m} M$ ), to improve the quality of impalements and to maximize the amount of neurotransmitter released. In many experiments we stimulated neurons with a high potassium solution. This solution consisted of the normal bathing solution $+150 \mathrm{~m} M \mathrm{KCl}$. The high potassium solution was applied from fine-tipped ( $2-5 \mu \mathrm{m})$ puffer pipets by pressure ejection. The duration of the pressure pulses was controlled electronically by a solenoid valve.

\section{Analysis of Synaptic Potential Amplitudes}

The amplitude of monosynaptic excitatory postsynaptic potentials (EPSPs) ranged from $<1 \mathrm{mV}$ to suprathreshold. The typical resting potential of sympathetic ganglion cell neurons was between $-40 \mathrm{mV}$ and $-60 \mathrm{mV}$, while the reversal potential for acetylcholine, the synaptic transmitter, was $-10 \mathrm{mV}$. Thus, differences in driving force from cell to cell, and nonlinear summation of the response to evoked transmitter release were both potential problems. Furthermore, repetitive stimulation at even moderate rates led to a large decrease in the EPSP amplitudes (see Fig. 2). For the purpose of analysis of EPSP amplitude versus days in co-culture (Table 1), cells were studied at their resting potential, and EPSP amplitude was considered to be the largest EPSP obtained after at least $30 \mathrm{~s}$ of inactivity. All suprathreshold EPSPs were assigned a value of $20 \mathrm{mV}$, which is likely to be an underestimate. However, since most EPSPs were subthreshold, the value used as an estimate of the size of the suprathreshold EPSPs had little effect on the mean EPSP amplitude at any of the time points. In some experiments the membrane potential of the postsynaptic neuron was set to $-90 \mathrm{mV}$, by passing constant hyperpolarizing current through the recording microelectrode. 
Table 1 (A) Proportion of Pairs of Preganglionic Neurons and Sympathetic Ganglion Neurons that were Monosynaptically Connected' and the (B) Proportion of Sympathetic Ganglion Cells in Co-Cultures that Received Excitatory Synaptic Inputs ${ }^{2}$

\begin{tabular}{cccccc}
\hline & \multicolumn{3}{c}{ A } & & B \\
\cline { 2 - 6 } $\begin{array}{c}\text { Days of } \\
\text { Co-Culture }\end{array}$ & $\begin{array}{c}\text { Percentage of Pairs } \\
\text { Monosynaptically } \\
\text { Connected }\end{array}$ & $\begin{array}{c}\text { Average EPSP } \\
\text { Amplitude }(\mathrm{mV}) \\
\pm \text { S.E.M. }\end{array}$ & $\begin{array}{c}\text { Number of } \\
\text { Pairs Tested }\end{array}$ & $\begin{array}{c}\text { Percentage of } \\
\text { Cells Receiving } \\
\text { EPSPs }\end{array}$ & $\begin{array}{c}\text { Number of } \\
\text { Cells Tested }\end{array}$ \\
\hline $1-1.5$ & 0 & - & 183 & 4.44 & 90 \\
$2-3$ & 3.0 & $13.1 \pm 3.7$ & 233 & 31.5 & 178 \\
$4-5$ & 1.74 & $11.1 \pm 2.5$ & 977 & 26.9 & 602 \\
$6-7$ & 2.86 & $9.2 \pm 2.0$ & 1013 & 25.9 & 764 \\
$8-9$ & 2.48 & $2.1 \pm 0.7$ & 202 & 20.7 & 150 \\
$10-12$ & 0.58 & 4.3 & 171 & 36.2 & 152 \\
\hline
\end{tabular}

${ }^{1}$ The criteria for determining whether a monosynaptic connection was present are described in the Results.

${ }^{2}$ The criteria for determining whether a neuron received synaptic input are described in Results. The number of pairs tesied for monosynaptic connections exceeded the number of ganglion cells tested for receiving EPSPs because there were often 1 wo or more preganglionic neurons in a single microscope field. As a consequence, for each ganglion cell impaled, several different potential synaptic connections could frequently be tested.

This prevented the larger EPSPs from reaching the threshold for spike initiation and enhanced our ability to detect small EPSPs.

Large EPSPs are not amenable to quantal analysis, due to non-linear summation (Martin, 1955). Analysis was therefore restricted to those connections at which the mean evoked synaptic potential was small $(<6 \mathrm{mV})$, and for which failures to release were present. In all cases analyzed in detail, the preganglionic neurons were stimulated at low frequency $(0.5 \mathrm{~Hz}$ or $0.2 \mathrm{~Hz})$, to avoid fatigue. The amplitudes of the monosynaptic EPSPs were measured semiautomatically. Data from the pre- and postsynaptic neurons were digitized at $5 \mathrm{kHz}$ beginning $10 \mathrm{~ms}$ before the stimulus to the presynaptic neuron and continuing for at least $190 \mathrm{~ms}$. A computer program identified the baseline and the point of the maximum depolarization that occurred in the postsynaptic neuron within $20 \mathrm{~ms}$ of the presynaptic spike (the peak of all monosynaptic EPSPs fell within this window). The baseline was obtained by averaging 10 points before the presynaptic spike, and the EPSP amplitude was calculated by subtracting the baseline from the average of five points straddling the maximum depolarization. The program displayed cursors for acceptance or rejection of the identified baseline and peak, and these cursors could be adjusted manually, if necessary, to eliminate artifacts. In particular, it was sometimes necessary to manually measure small EPSPs, because the capacitative coupling artifact due to stimulation of the presynaptic neuron caused a larger depolarization than the evokcd EPSP. In all cases in which the computer-estimated peak response was negative, the response was assigned a value of $0 \mathrm{mV}$.

We analyzed evoked synaptic potential amplitudes both with and without correction for non-linear summation (Martin, 1955) and for drift in the resting potential. Data were normalized to a resting potential of $-50 \mathrm{mV}$, and the reversal potential for the synaptic potentials was taken to be $-10 \mathrm{mV}$. The data illustrated in the Figures are corrected data, but analysis of the uncorrected and the corrected data led to the same conclusions.

The distribution of delayed release EPSPs was fit to Equation 1 of Bekkers, Richerson, and Stevens (1990) or to the sum of a series of gaussian distributions. The amplitudes of delayed release EPSPs were put into $0.1-\mathrm{mV}$ bins, and the histograms of these bins were fit by a nonlinear curve fit program (Sigmaplot 4.0). The gaussian fits were subject to the constraints that the standard deviation of all peaks had to be at least as great as the background recording noise, that the peaks had to be separated by at least threc times the standard deviation of the background noise, and that each peak represent at least $5 \%$ of the total area. In practice, this meant that any peaks less than $.3 \mathrm{mV}$ apart would be treated as a single peak.

The histograms of monosynaptic EPSPs were not fit to a theoretical distribution for reasons explained in Results. However, we performed numerical simulations to gain insight as to how much variation in the size of individual quanta would be necessary to obscure the expected peaks in the histogram of evoked EPSPs, or to create artifactual peaks. For these simulations we created model data sets of 180 EPSPs (the mean number of EPSPs in the real data sets we analyzed). Each model data set was created by using a random number generator to draw values from a Poisson distribution. In the simulation program, we could specify the mean quantal content $(m)$, the coefficient of variation of the unitary response ( $\mathrm{CV}=$ standard deviation/mean), and the signal to noise ratio ( $\mathrm{SN}=$ mean quantal response amplitude/standard deviation of the baseline noise). However, we held $S N$ constant at its mean value of 6.5 , and varied $m$ and $\mathrm{CV}$. The parameter $\mathrm{m}$ was allowed to range between 0.5 and 3.5 (in increments of .5 ), because it is over this range that one would expect to observe a signifi- 
cant number of failures, and of single and double quantal releases. For each value of $\mathrm{m}, \mathrm{CV}$ was gradually increased (from .10 in increments of .05) until peaks at the levels expected for single and double releases could be detected by visual inspection in fewer than $50 \%$ of the simulations. Five to ten simulations were run for each set of parameters.

A possible concern of using a high-calcium recording solution is that it might tend to promote conduction block in some axonal branches. This would distort the evoked monosynaptic EPSP histograms by giving more failures and fewer single and double quantal responses than expected. However, conduction block would not be expected to alter the location of peaks in the histogram, and therefore it should not have had any effect on the analysis reported.

\section{RESULTS}

The preganglionic neurons and sympathetic ganglion neurons that we studied in vitro were obtained from chick embryos shortly after the stages at which they first begin to encounter each other in vivo (Yip, 1987). Cells were plated at a density of about 1 preganglionic neuron and 10 ganglion cells per $450-\mu \mathrm{m}$ diameter microscope field. The preganglionic neurons initiate process outgrowth within a few hours of plating (Honig and Hume, 1986 ) and axonal outgrowth proceeds at a rate of $40-45 \mu \mathrm{m} / \mathrm{h}$ (Moorman and Hume, 1990), so that the initial physical contacts between preganglionic neurites and sympathetic ganglion neurons were most likely made within $24 \mathrm{~h}$ after plating. However, the time of initial interaction for any particular pair of cells was unknown.

\section{Frequency of Occurrence of Synaptic Connections}

We made intracellular recordings from 1936 sympathetic ganglion neurons in co-culture with preganglionic neurons. The most direct way to assess the frequency of occurrence of synaptic connections would have been to simultaneously penetrate preganglionic neurons and ganglion cells with separate microelectrodes for intracellular stimulation and recording. However, we quickly discovered that it was very difficult to make stable intracellular recordings from preganglionic neurons until they had been in culture for 4-5 days. Furthermore, we found that synaptically connected pairs of cells were relatively rare (see below). For these reasons, we developed a method that allowed us to rapidly test whether pairs of cells were synaptically connected without having to penetrate the preganglionic neurons. We made intracellular recordings from sympathetic ganglion neurons, and then stimulated the cell body of each preganglionic neuron in the microscope field with a brief application ( $50 \mathrm{~ms}$ ) of high-potassium solution ejected from a puffer pipet. If no EPSP was seen, then the pair was scored as not synaptically connected. The stimulation by high potassium evoked spikes in $>95 \%$ of preganglionic neurons studied in experiments designed to evaluate this method, so this method of stimulation produced few false-negatives. Briefly, if high-potassium stimulation of a preganglionic neuron evoked an excitatory postsynaptic potential in a nearby sympathetic ganglion neuron, then the pair was scored as possibly connected. For each such pair, we then attempted to determine whether the cells were synaptically connected, or whether a presynaptic fiber running near the preganglionic neuron cell body had been stimulated by the highpotassium solution. We made intracellular penetrations of each candidate presynaptic cell and then stimulated the preganglionic neuron to see if it could evoke EPSPs in the ganglion cell under study. Besides being quick, the high-potassium stimulation paradigm was also quite accurate. In 53 of the 74 cases where a short puff of high-potassium solution to a preganglionic cell body elicited an EPSP in a nearby ganglion cell, subsequent intracellular stimulation showed that the pair was monosynaptically connected. The 21 cases of failure to confirm monosynaptic connections included 12 cases where we failed to make an adequate penetration of the putative presynaptic neuron, as well as nine false-positives in which preganglionic spikes did not evoke EPSPs. Evoked synaptic potentials were considered to be monosynaptic only in cases in which intracellular stimulation produced synaptic potentials at a short, nearly fixed (less than $1 \mathrm{~ms}$ fluctuations) latency [Fig. 1(A)]. In addition to the monosynaptic responses, at many synapses there were also synaptic potentials occurring at irregular intervals in the first few hundred milliseconds after the peak of the monosynaptic EPSP [Fig. 1 (B)]. We observed no spontaneous synaptic potentials in the absence of stimulation, so these delayed release EPSPs were also a consequence of presynaptic stimulation. The significance of these delayed release EPSPs is considered below.

Monosynaptic EPSPs from preganglionic neurons to sympathetic ganglion neurons were not detected at 24 or $36 \mathrm{~h}$ of co-culture, but monosynaptic EPSPs were seen by $48 \mathrm{~h}$ of co-culture [Table 

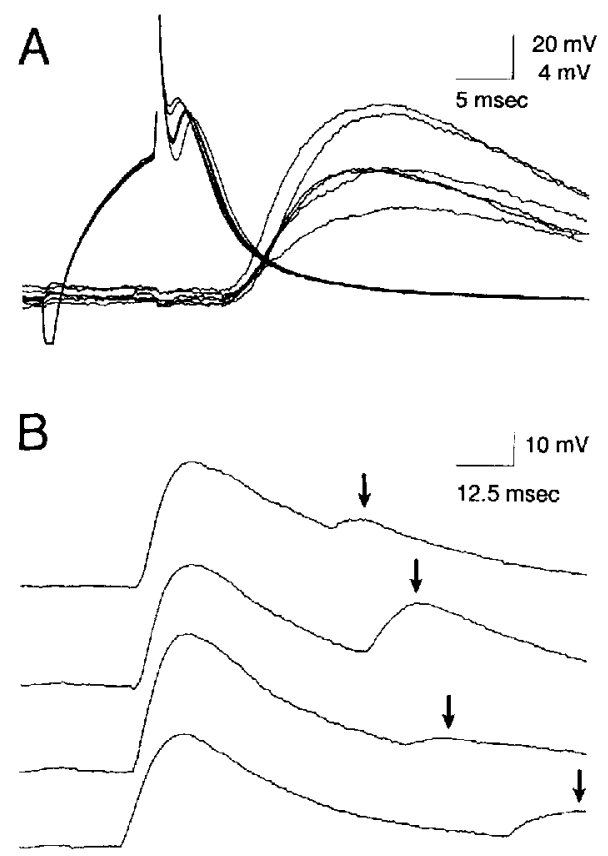

Figure 1 (A) Properties of a monosynaptic connection from a preganglionic neuron to a ganglion cell. The spike elicited in the preganglionic neuron by current injection (for the duration indicated by the stimulus artifacts) was followed at short latency by an EPSP in the sympathetic ganglion neuron. Six successive trials are illustrated. Note that the EPSP amplitude fluctuated from trial to trial. (B) Examples of delayed release EPSPs (arrows) that often occurred on the falling phase of the monosynaptic EPSPs.

1(A)]. However, only a small proportion of the preganglionic neurons that we tested (about $2 \%$ ) evoked EPSPs in ganglion cells in the same microscope field. The incidence of monosynaptic EPSPs did not change dramatically over the time period of 2-12 days [Table 1(A)], though there may have been a slight decline at 10-12 days. Furthermore, we detected no tendency for the amplitude of the evoked synaptic potentials to increase in older cocultures. In fact, the monosynaptic EPSPs in the older co-cultures on average were somewhat smaller than those studied in younger co-cultures. These results suggest that functional synapses do not form until about 2 days of co-culture, and that following the initial burst of synaptogenesis either no additional synapses form, or the rates of formation and elimination of synapses are similar.

One possible alternative explanation for the failure to observe evoked EPSPs before 2 days was that before this time the preganglionic neurons were not capable of generating action potentials. However, it is quite easy to make whole-cell patch- clamp recordings from preganglionic neurons at all times after they are placed into culture (Clendening and Hume, 1990). Using this technique we found that application of the high-potassium solution to preganglionic neurons in culture for 1 day evoked spikes in $100 \%$ of the cells tested $(n=10)$. We chose not to use whole-cell recording from preganglionic neurons for routine screening of synaptic connections, because we were concerned that the internal dialysis that accompanies this type of recording might disrupt the ability to release neurotransmitter. In particular, it is well established that many voltage-dependent calcium currents rapidly disappear with whole-cell recording (Fenwick, Marty, and Neher, 1982).

Because we were surprised by the slow onset of synaptic transmission, and by the lack of a developmental increase in the frequency of synaptic connections, we estimated the time course of synapse formation in a second way. We determined how many sympathetic ganglion neurons in co-cultures of various ages received excitatory synaptic inputs from any source. Long applications ( $1 \mathrm{~s}$ ) of the high-potassium solution stimulated most or all of the presynaptic axons passing through about onequarter of the microscope field. Thus, by recording from ganglion cells and then making puffs in several different positions we could determine whether each ganglion cell received innervation from any source, including neurons whose cell bodies were not in the same microscope field. As was the case with the proportion of pairs that were monosynaptically connected, the proportion of ganglion cells that received innervation increased dramatically after about 2 days of co-culture (to about $25 \%-30 \%$ ), and then neither increased nor decreased greatly between days 2 and 12 of co-culture [Table 1(B)]. However, in contrast to the results with monosynaptic connections, some ganglion cells (about $5 \%$ ) were shown to already receive synaptic potentials at $24 \mathrm{~h}$ of co-culture. A concern with this indirect method was that one could not be sure that the synaptic potentials recorded in ganglion cells were due to the action of the preganglionic neurons. For instance, it is well known that rat sympathetic ganglion neurons can make cholinergic synapses onto each other when the culture medium contains factors that promote the cholinergic differentiation of the neurons (O'Lague, Obata, Claude, Furshpan, and Potter, 1974). Therefore, it was important to determine the frequency with which EPSPs could be elicited in chick sympathetic ganglion neurons grown in culture without other neurons. When our standard 
medium, which contained no embryo extract or muscle-conditioned medium, was used, sympathetic ganglion neurons grown alone formed excitatory synapses with each other at low frequency. Only $8 \%$ of sympathetic ganglion neurons in culture alone received EPSPs in response to high-potassium stimulation ( $5-28$ days, $n=95$ ). Thus, the presence of spinal cord cells resulted in a three- to fourfold increase in the proportion of ganglion cells that received EPSPs. It should be noted that the proportion of neurons receiving EPSPs in the control, sympathetic ganglion neuron alone, cultures was actually higher than the proportion of ganglion cells receiving EPSPs in 1 day co-cultures [Table 1(B)]. Thus cholinergic synapses formed between ganglion cells, rather than synapses between preganglionic neurons and ganglion cells, probably account for the synaptic potentials that could be evoked before 2 days of co-culture. In summary, both approaches yielded similar information about the time course of synapse formation between preganglionic neurons and sympathetic ganglion neurons in this culture system.

\section{PHYSIOLOGICAL PROPERTIES OF THE SYNAPSES MADE BY PREGANGLIONIC NEURONS ONTO GANGLION CELLS}

When a presynaptic preganglionic neuron was stimulated for a series of trials, the monosynaptic responses recorded in the postsynaptic ganglion cell fluctuated considerably from trial to trial [Fig. 1(A)]. Furthermore, when presynaptic neurons were stimulated repetitively, even at moderate rates, there was an obvious decrease in the amplitude of synaptic potentials. This was particularly striking for monosynaptic EPSPs that were initially quite large (Fig. 2). In this example, the initial EPSP of each trial (with the cell held at $-90 \mathrm{mV}$ by hyperpolarizing current) was between 25 and 40 $\mathrm{mV}$. By the fourth spike of a train of stimuli at 2 $\mathrm{Hz}$, occasional stimuli failed to release any transmitter, and within 10 spikes, the mean amplitude of the monosynaptic EPSPs had declined to a steady state of only $5 \mathrm{mV}$. In order to record a series of synaptic potentials that had the same mean amplitude at the beginning and end of the trial, it was necessary to stimulate at no higher than $0.5 \mathrm{~Hz}$. For some synapses (like the one illustrated in Fig. 2) it was necessary to decrease the stimulus rate to $0.2 \mathrm{~Hz}$ to prevent a decline in the amplitude of the EPSPs.

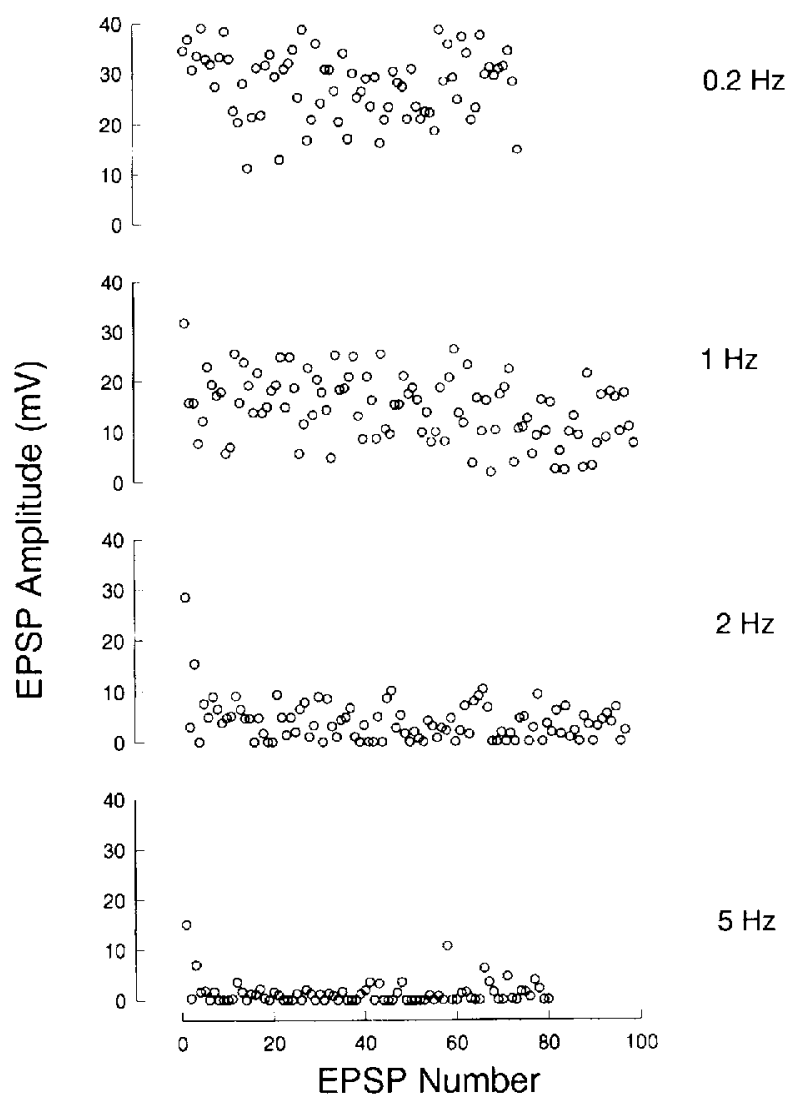

Figure 2 Monosynaptic EPSPs depressed with repetitive stimulation at low frequency. All data are from a single pair of neurons. EPSPs were evoked by intracellular stimulation of the preganglionic neuron at the rates indicated. Extensive trial to trial fluctuations in the amplitude of the EPSP were apparent at all stimulus frequencies. At a stimulus frequency of $0.2 \mathrm{~Hz}$ the average amplitude of the EPSP was stable over time, but at $1 \mathrm{~Hz}$ or higher the EPSPs were, on average, smaller at the end of the run than at the beginning. There was also some depression that carried over between trials, as indicated by the decline in the first EPSP of the train over trials. The data at $0.2 \mathrm{~Hz}$ were taken first, those at $1 \mathrm{~Hz}$, second, those at $2 \mathrm{~Hz}$, third and those at $5 \mathrm{~Hz}$, last. The potential of the postsynaptic neuron was set to $-90 \mathrm{mV}$ by passing hyperpolarizing current through the recording electrode.

To gain insight into the way that synaptic transmission functions at these recently formed synapses, we decided to analyze the pattern of fluctuations in synaptic potentials in more detail. However, the difficulty of maintaining impalements in these small cells, and the requirement that we stimulate only at low frequency meant that we were only able to gather a limited amount of information from each pair. For this reason, we were unable to perform an analysis as detailed as is possible at mature synapses. 
The quantal hypothesis postulates that transmitter is released in multimolecular packets of relatively fixed size (quanta), and that evoked responses consist of the synchronous release of an integral number of quanta (del Castillo and Katz, 1954). A prediction of this hypothesis, which has been verified at many mature synapses, is that under conditions where the mean level of release is low, and the background noise is not too large, the amplitude distribution of monosynaptic EPSPs should consist of an initial peak at $0 \mathrm{mV}$, representing failure to release any transmitter, and then a series of equally spaced peaks that represent the release of 1 and 2 quanta. The relative number of events that contribute to each peak is determined by some particular statistical rule (e.g., Poisson, binomial, compound binomial, reviewed in Redman, 1990) but for low levels of release all these statistical rules give rise to distributions with evenly spaced peaks. A second manifestation of the quantal nature of the release process at the neuromuscular junction is the phenomenon of delayed release (Liley, 1956; Rahamimoff and Yaari, 1973). Studies at the frog neuromuscular junction suggest that delayed release is a consequence of an elevation in the free-calcium level in the nerve terminal that persists for a short time after each spike. This residual calcium is high enough to enhance the probability that individual quanta will be released, but low enough that the release is asynchronous. For this reason, it is generally thought that each delayed release FPSP is likely to represent the effect of a single quantum. However, at synapses between preganglionic axons and autonomic neurons there is substantial evidence that some of the delayed release EPSPs are due to the simultaneous release of two or more quanta (Martin and Pilar, 1964; Dennis et al., 1971; Bornstein 1974; 1978).

We analyzed the amplitude of both types of evoked synaptic potentials, monosynaptic EPSPs and delayed release EPSPs. The amplitude of the monosynaptic EPSP was taken to be the maximum depolarization from baseline reached within the initial $20 \mathrm{~ms}$ following the onset of the synaptic response. Delayed release EPSPs were measured in a $150-\mathrm{ms}$ time interval beginning $20 \mathrm{~ms}$ after the peak of the monosynaptic EPSP. In many cases, the delayed release EPSPs occurred during the falling phase of the monosynaptic EPSP, so they were superimposed on a falling baseline. To correct for the slope of the baseline, we fit an exponential curve by eye to the falling phase of the EPSP just before the delayed release occurred. The difference between the exponential curve and the membrane potential at the peak of the EPSP was taken to be the amplitude of the delayed release event.

\section{Analysis of Delayed Release EPSPS}

Before we analyzed the properties of delayed release EPSPs in detail, it was important to eliminate the possibility that the delayed release EPSPs represented polysynaptic EPSPs rather than the delayed release of transmitter from the terminals of the preganglionic neurons. In particular, the preganglionic neuron might also have synapsed on a second ganglion cell (an "interneuron") that made a cholinergic synapse onto the ganglion cell from which the recordings were made. Several lines of evidence argue against this possibility. First, the overall incidence of synaptic connections among ganglion cell was quite low in these cultures (see above). Therefore, it would have been unexpected for polysynaptic connections to be common, yet delayed release EPSPs were present at over $50 \%$ of the synapses studied. Second, the latency from the peak of the presynaptic spike to the onset of the delayed release event was often quite long (>150 ms) and varied widely from trial to trial. If the preganglionic neuron made a suprathreshold connection to an "interneuron" that then synaptically excited the ganglion cell under study, then the "interneuron" spike would have been expected to be initiated on the rising phase of the EPSP. If we assume the EPSPs in the "interneuron" were no different than in any other ganglion cell, then the delay to the onset of a disynaptic EPSP should have been only a few ms, and the delayed EPSP should have showed latency fluctuations not much greater than the rise time of an EPSP ( $<20 \mathrm{~ms}$ ). By these criteria only one of the 53 pairs of cells had a pattern of responses that suggested that a polysynaptic EPSP might be present, and too few EPSPs were obtained from this pair for analysis. Third, the amplitude of the delayed release EPSPs typically were near the lower end of the range of monosynaptic EPSP amplitudes, as would be expected if they were the quantal components from which the monosynaptic EPSPs were built. Thus it seems likely that the delayed release EPSPs were the result of transmitter release from the terminals of the preganglionic neurons.

We collected a sufficient number of delayed release EPSPs for analysis at eight synapses. In three of these eight cases [Fig. 3(A-C)] the distribution of delayed release EPSPs was well described by a single gaussian function with a coefficient of varia- 

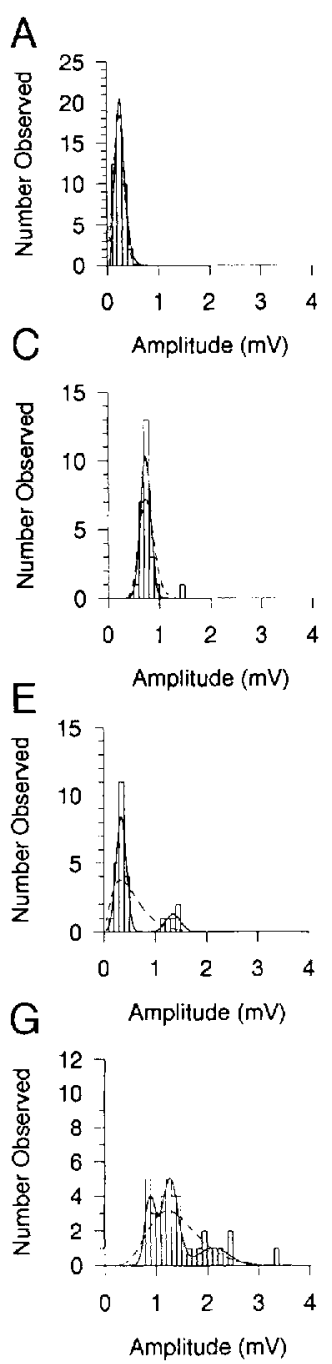

Figure 3 Histograms of the amplitudes of delayed release EPSPs. Each panel is the set of responses for a different synapse. The dashed curves are the fit of the data to a continuous distribution described by Equation 1 of Bekkers et al. (1990). The solid curves are the sum of the gaussian functions that were required to fit the data subject to the constraints described in Methods. The mean and standard deviation of the gaussian curves used in the fits were (A) $0.24 \pm .07$; (B) $0.36 \pm .1 ;$ (C) $.73 \pm .1$; (D) $.4 \pm .1, .9 \pm .11$; (E) $.34 \pm .1,1.35 \pm .14$; (F) $.93 \pm .19$, $1.5 \pm .14,1.8 \pm .1 ;(\mathrm{G}) .91 \pm .12,1.29 \pm .15,1.93 \pm .12$, $2.4 \pm .12 ;(\mathrm{H}) .51 \pm .06, .75 \pm .08,1.26 \pm .12,2.15 \pm .25$, $2.8 \pm .24$.

tion ( $\mathrm{CV}=$ standard deviation / mean ) of less than 0.3 . However, for the other five pairs of neurons studied [Fig. 3(D-H)], the amplitude distributions for delayed release EPSPs were extremely broad $(\mathrm{CV}=.4-.76)$, and there appeared to be discrete peaks in the distribution. The delayed release events we studied at these five developing neu- ronal synapses had an amplitude distribution very different from that for single quantal responses observed at mature neuromuscular junctions. The amplitude distribution of spontaneous releases at mature neuromuscular junctions is typically unimodal, with a CV of 0.2-0.3 (Fatt and Katz, 1952; Boyd and Martin, 1956).

The broad amplitude distribution of delayed EPSPs can be interpreted in two quite different ways. One possibility is that these delayed EPSPs were sampled from continuous distributions that had a large intrinsic variance. In this case, the peaks in the histograms would represent an artifact of the small sample size. Alternatively, the histograms of delayed EPSPs might have been broad because they represent the sum of a number of distinct distributions, each of which has relatively little intrinsic variance.

Unitary quantal events with a large intrinsic variance have been described elsewhere. For example, in hippocampal neurons miniature synaptic currents seem to be drawn from a continuous distribution with a CV of about 0.55 (Bekkers et al. 1990). These authors presented an equation that describes the expected distribution of events when a random process has a large intrinsic variance. When we fit our data with this equation [Fig. 3 (dashed lines)] the curves did not closely follow the data points, but a $\chi^{2}$ test showed that the fits could not be rejected. However, if delayed EPSPs at the synapses we studied were drawn from a continuous distribution, it is surprising that peaks were observed in all five cases with a large coefficient of variation.

For all five of the synapses that showed a large coefficient of variation, the pattern of delayed release EPSPs could be very well fit by the sum of two or more distinct gaussian distributions, each of which had a coefficient of variation of less than 0.3 [Fig. 3 (solid lines)]. Very broad distributions for delayed release EPSPs, which could be fit by the sum of gaussian functions, have been described for mature synapses between preganglionic axons and some autonomic neurons (Blackman et al. 1963a; Martin and Pilar, 1964; Dennis et al., 1971; Bornstein $1974 ; 1978$ ). However, the broad distributions of delayed release EPSPs we observed at newly formed synapses appeared to differ in one important respect from those described at mature synapses. At the mature synapses described by others, the histograms of delayed release EPSPs usually had obvious peaks that were at integral multiples of the smallest EPSPs. To explain this observation, it was suggested that the larger responses 
consisted of the synchronous release of more than one quantum, but that quantal size was relatively constant. In contrast, for all five synapses we studied, the apparent peaks were not at integral multiples of the smallest EPSPs. This is particularly apparent in Fig. 3(E), where one peak occurred at $0.35 \mathrm{mV}$ and a second peak at $1.35 \mathrm{mV}$, with no indication of events of intermediate amplitude. A possible explanation of this result is that quanta of different sizes were present at this newly formed synapse.

The presence or absence of a large coefficient of variation in the histograms of delayed releases was not related to the length of time that the cells had been in co-culture. Histograms of delayed release EPSPs with multiple peaks were found for cells in co-culture for 4-8 days, while histograms with a single sharp peak were obtained from cells in coculture from 6 to 8 days. Despite this result, the possibility that synapses having delayed releases of a single size class are more mature (or less mature) than those with multiple size classes cannot be completely eliminated. We do not know the time of initial synapse formation for each pair, so it is possible that some of the synapses studied at later times of co-culture had not formed until several days after others in the same dish.

One possible factor that could lead to dispersion in the amplitude of quantal events is electrotonic decrement. If one release site was on the cell body and another on a distal process that was quite long, then the two sites might have been electrically distant enough for them to have unequal effects. We did not observe large differences in the shape of EPSPs, as might have been expected if there was considerable electrotonic dispersion. In a further attempt to test whether the synapses from a single preganglionic neuron can be widely dispersed, we tried to examine the properties of synaptic transmission in a series of cells in which we had intracellularly stained both the pre- and postsynaptic cells, so that we could determine the actual sites of synaptic contact. This proved quite difficult, but a few such pairs were obtained. For cells that were synaptically connected, we always saw apparent contacts between branches of the preganglionic axon and the surface of the postsynaptic ganglion cell ( $n$ $=6$ ). These contacts were characterized by bouton-like enlargements (Fig. 4), and they were on either the ganglion cell body alone $(n=2)$, or on both the dendrites and cell body $(n=4)$. For the pair illustrated in Figure 4, all seven boutons were on the surface of the sympathetic ganglion neuron cell body, thus for this particular synapse electro- tonic factors should not have varied among release sites. Unfortunately, not enough EPSPs were collected from any pair for which we obtained anatomical data to perform an analysis of EPSP fluctuations. However, in all the cases in which we intracellularly stained ganglion cells in culture $(n=12)$ the dendritic arbors were quite modest. In fact, the sympathetic ganglion neuron shown in Figure 4 had relatively long dendrites, with three of its four dendrites being longer than average. The average length of the dendrites was $50.2 \mu \mathrm{m}$ and only three of 36 dendrites were longer than $100 \mu \mathrm{m}$ (the longest dendrite was $148 \mu \mathrm{m}$ ). The average sympathetic ganglion neuron had three dendrites (range: $0-7$ ). Because the dendrites were short, and the cell bodies quite large (mean cell body diameter: $37 \mu \mathrm{m}$ ) it seems unlikely that electrotonic factors were a significant cause of the dispersion in amplitudes of components of the synaptic responses.

\section{Analysis of Monosynaptic EPSPs}

Analysis of the amplitude distribution of monosynaptic EPSPs provided additional information about transmission at these recently formed synapses. When a significant number of failures are present, it is statistically likely that many of the releases will consist of one or two quanta. If the background noise is low and the intrinsic variance of the quantum is small, then there should be evenly spaced peaks in the amplitude histogram corresponding to release of 1 and 2 quanta. For 8 of the 12 synapses we studied, there were a significant number of trials in which a presynaptic spike elicited no detectable response (a detectable response was defined as $>0.2 \mathrm{mV}$ ). We consider most of these events to be genuine failures rather than very small responses since (1) the bin next to the failures $(0.2-0.4 \mathrm{mV})$ was nearly devoid of events in seven of the eight cases and (2) the amplitude of small delayed EPSPs (which are presumably responses to single quanta) was seldom smaller than $0.4 \mathrm{mV}$. The one case in which failures could not be clearly distinguished from small responses [Fig. $5(\mathrm{~A})]$ was from a synapse where the delayed EPSPs were unusually small [ mean $0.24 \mathrm{mV}$, Fig. $3(\mathrm{~A})$ ], and so no gap between failures and small events was expected.

Histograms of monosynaptic EPSPs with evenly spaced peaks have been described for synaptic transmission at synapses in mature autonomic ganglia (Blackman et al. 1963b; Martin and Pilar, 1964; Dennis et al., 1971; Bornstein, 1974). However, evenly spaced peaks were not apparent in the 


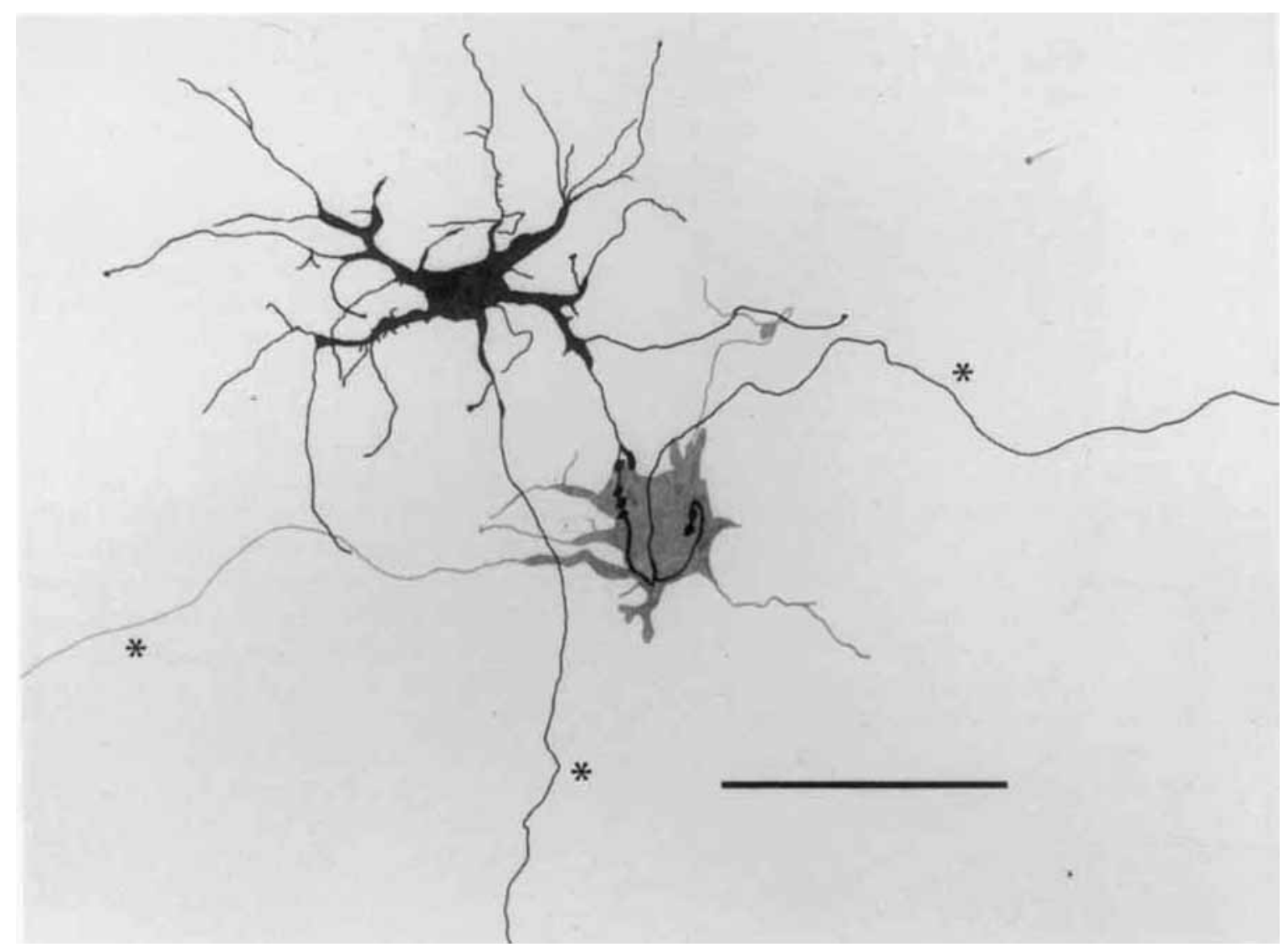

Figure 4 Camera lucida drawing of a synaptically connected pair of neurons. The 27 monosynaptic EPSPs recorded at this synapse ranged from 2.2 to $10.2 \mathrm{mV}$. Both the presynaptic preganglionic neuron (black) and the postsynaptic sympathetic ganglion neuron (gray) were stained by intracellular injection of horseradish peroxidase. To allow us to see presynaptic boutons contacting the ganglion cell soma, we used a brief duration of injection into the ganglion neuron so that it was only lightly stained. Seven swellings of the terminal branches of the preganglionic axon were visible on the surface of the ganglion cell body. There were no other apparent sites of contact. This preganglionic neuron was unusual in that it had two axons (asterisks), although only one contacted the stained ganglion cell. The sympathetic ganglion neuron had a single axon (asterisk), and short, simple dendrites. All axons traveled for many hundred $\mu \mathrm{m}$ beyond the points illustrated. Scale bar $=100 \mu \mathrm{m}$.

histograms of monosynaptic EPSPs we obtained [with the possible exception of Fig. 5(F)]. Some histograms had no obvious peaks [Fig. 5(A)], and others had apparent peaks at nonintegral amplitudes [Fig. 5(D)]. However, the apparent peaks were so narrow that they are as likely to have arisen from statistical fluctuations due to the relatively small size of the samples as to represent real peaks.

The implication of these results is that the variance associated with our recordings was probably too great to allow us to distinguish true peaks in the histograms of monosynaptic EPSPs. One way this could have come about is if quanta come in a number of discrete sizes, since in this case, one would not expect to find equally spaced peaks in the his- tograms. Alternatively, all quanta may come from a single continuous distribution, but the background noise or the intrinsic variance of the quanta may have obscured the peaks of the histogram. The standard deviation of the background noise averaged $0.083 \mathrm{mV}$, and the mean size of the first peak in the histograms of delayed releases (which provides a minimum estimate of quantal size) was $0.55 \mathrm{mV}$. With this level of background noise, our numerical simulations (see Methods) indicated that if quanta come from a single continuous distribution, then the coefficient of variation of the quantal responses must be greater than 0.25 . Were the coefficient of variation any less, then more than half of the histograms would have been expected to 
A
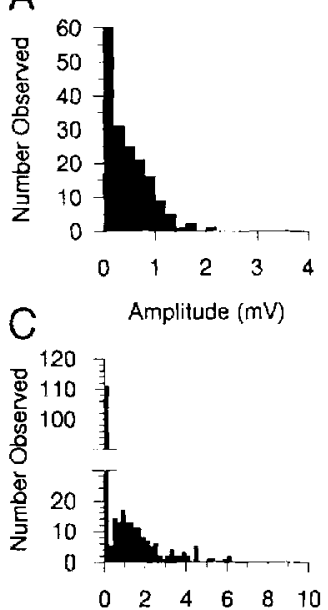

$\begin{array}{llllll}0 & 2 & 4 & 6 & 8 & 10\end{array}$
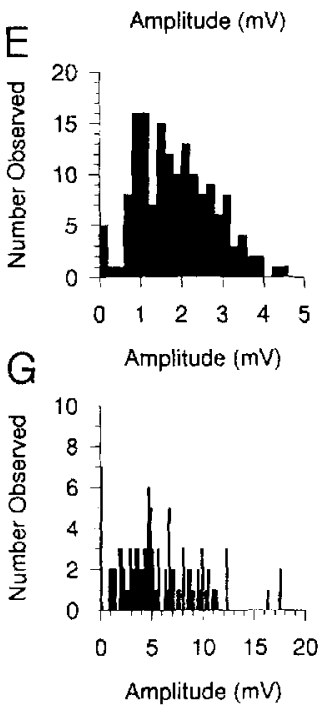

$\mathrm{B}$
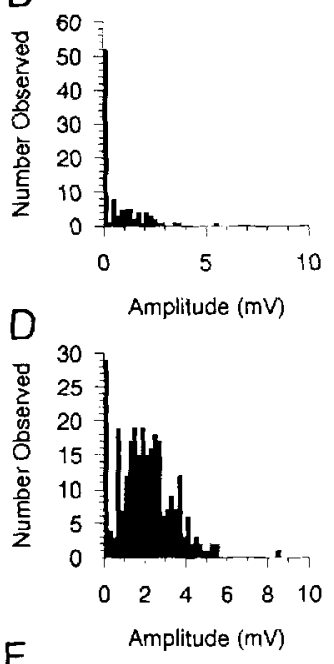

F

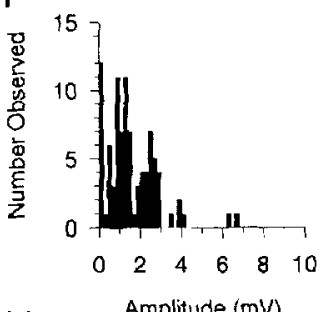

H

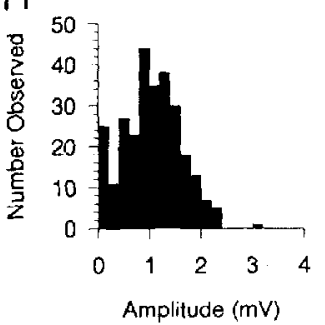

Figure 5 Histograms of the amplitudes of monosynaptic EPSPs. Each panel is data from a different synapse. The mean monosynaptic EPSPs recorded at all of these synapses were less than $6 \mathrm{mV}$, and there were failures on at least some of the trials. The preganglionic neurons were stimulated intracellularly at $0.2 \mathrm{~Hz}$ or $0.5 \mathrm{~Hz}$, and no decline in monosynaptic EPSP amplitude was observed over the course of stimulation. The data in panels $A, D, E$, and $F$ are from the same synapses as the delayed release data shown in the corresponding panels of Figure 3. Too few delayed EPSPs were obtained for the other four synapses shown in this figure for analysis of delayed EPSPs to be made. The monosynaptic EPSP histograms for the synapses corresponding to Panels $\mathrm{B}, \mathrm{C}, \mathrm{G}$, and $\mathrm{H}$ of Figure 3 were not analyzed in detail, because the average responses were too large to be amenable to analysis, and because there were few, if any failures. The mean EPSP amplitude for the four synapses that are not illustrated were $10.3,9.1,15.0$, and $18.1 \mathrm{mV}$, respectively.

show obvious peaks corresponding to the release of 1 and 2 quanta. These results provide evidence that the monosynaptic EPSPs at these synapses cannot

consist of the summation of quanta of very low intrinsic variance, as has been suggested to be the case at some synapses in the central nervous system (Jack, Redman, and Wong, 1981). The quantal responses also appear to have more intrinsic variance than those reported in some autonomic ganglia (Bornstein, 1974). In conclusion, our analysis of the amplitudes of monosynaptic EPSPs is consistent with our observations on delayed release EPSPs, since both suggest that the coefficient of variation of quantal responses is relatively large.

At mature synapses, the number of monosynaptic EPSPs of each amplitude class type can usually be well described by a statistical rule, such as the Poisson, binomial, or compound binominal rule (Redman, 1990). Because we were unsure about the amplitude distribution of the single quantal events, it seemed unwise to fit a theorctical distribution to the monosynaptic EPSP data. In particular, if quanta come in different size classes, there is no basis for deciding whether all size classes of quanta have the same probability of release, or what fraction of the quanta belong to each size class. However, even without knowing the amplitude distribution of single quantal events, limits can be put on some of the parameters that govern transmitter release. First, an estimate for $n$, the number of sites capable of releasing transmitter, can be obtained by dividing the largest evoked EPSP seen at each synapse by the largest delayed EPSP seen at the same synapse (A). This calculation assumes that the monosynaptic EPSP is made up of quanta equal to or smaller than the largest delayed EPSPs, and will underestimate $n$ if $p$, the probability of releasing a quantum, is small, and will overestimate $n$ if the largest quanta are not represented among the sample of delayed release EPSPs. $n$ was estimated to be 10 or less for 6 synapses, was estimated to be 24 for 1 synapse, and was estimated to be 80 for 1 synapse. With the exception of the synapse with an estimated $n$ of 80 , this range of $n$ is in good accord with the range of stained boutons (3-30) we observed at doubly labeled pairs of neurons. Second, an upper limit to $p$ can be calculated. Since the mean EPSP is expected to equal $n^{*} p^{*} \mathrm{~A}$, and the maximal EPSP can be no larger than $n^{*} \mathrm{~A}$, then $p$ should not exceed the mean EPSP/maximal EPSP, whether or not the actual values of $A$ and $n$ are known. For the twelve synapses we studied, the estimated upper limit for $p$ was $<.25$ for six cases, and was $>.5$ for only three cases. The low estimate of $p$ is somewhat surprising given that we had a relatively high extracellular calcium concentration $(5.4 \mathrm{~m} M)$. However, a low $p$ value is consistent with the observation of failures. 


\section{DISCUSSION}

In this study we show that sympathetic preganglionic neurons can form functional synapses in culture with their normal target cells ( sympathetic ganglion neurons). However, only in about $2 \%$ of the pairs tested was a preganglionic neuron monosynaptically connected to a nearby ganglion cell. The low incidence of connected pairs of cells is likely to be, at least in part, a consequence of the culture system, and has no direct implications as to the ease with which these cells can form synapses. We allowed neurons to settle randomly throughout the culture dish, and tested all the ganglion cells that were in the same field of view as the preganglionic neuron. Most preganglionic neurons have only a single axon (Honig and Hume, 1986); consequently cells that are in the same field do not always have an opportunity to make synaptic contact (Nelson et al., 1983). For instance, if a ganglion cell were $10 \mu \mathrm{m}$ to the right of a preganglionic neuron, but the preganglionic axon grew out of the left side of the cell, then the two cells would probably never encounter each other, unless there were some mechanism that attracted axons from a distance. The large number of cases in which the axon of a preganglionic neuron came frustratingly close to a particular ganglion cell without contacting it suggests that no such attraction operated in our cultures. In addition, the axons of preganglionic neurons could grow for several millimeters, so the high overall proportion of ganglion cells that receive innervation from somewhere in the dish (about 25\%) is not inconsistent with the low incidence of monosynaptic connections within a field of view.

Although the simplest explanation of our results is that the low frequency with which we found monosynaptically connected pairs was the result of the relatively low probability of encounter in these mass cultures, an additional possibility is worth considering. There may be a mechanism that ensures that there is only a low probability of synapse formation between a preganglionic neuron and any particular ganglion cell it encounters. Results from studies of the organization of mature mammalian autonomic ganglia suggest that in vivo, each preganglionic neuron ramifies widely throughout a large territory, and yet makes synapses with only a relatively small proportion of the target cells around it (Hume and Purves, 1983). A low probability of synapse formation would be one way to achieve effective dispersal. The best information available on the probability of forming a synapse comes from elegant studies of neuromuscu- lar junctions in culture. These studies indicate that functional synapses form with high probability within a few seconds after motor neurons contact muscle (Kidokoro and Yeh, 1982; Sun and Poo, 1987; Evers, Laser, Sun, Xie, and Poo, 1989). The hypothesis that the probability of synapse formation is low predicts that pairs of preganglionic neurons and ganglion cells studied with similar methods would produce a quite different result.

Our results suggest that the responses to individual quanta varied substantially. Variation in the response to individual quanta could arise in a number of ways, including variation in the number of molecules of acetylcholine per quantum, and in the distance from the release site to the postsynaptic cell (Evers et al., 1989). Electrotonic factors are unlikely to be the cause of the variation, since the dendritic arbors of the sympathetic ganglion neurons are so small. One attractive explanation for our results is that the density and type of acetylcholine receptors varies among the different synaptic sites on the surface of developing sympathetic ganglion neurons. Role (1988) showed that sympathetic ganglion neurons taken from early embryos produce only modest currents in response to acetylcholine when grown alone, and that acetylcholine responsiveness is increased tenfold in sympathetic ganglion neurons that receive EPSPs from spinal cord explants. More recently, Role and her collaborators (Moss, Scheutze, and Role, 1989) showed that chick sympathetic ganglion neurons express several different types of acetylcholine receptor channels, and that as development proceeds, openings with large unitary conductances and relatively long open times become more common. We have confirmed some of these results (Walker and Hume, 1990) by making whole-cell recordings from sympathetic ganglion neurons taken from stage 35 embryos, and then grown in culture without other cells. Under our culture conditions, the peak whole-cell currents elicited in response to saturating levels of acetylcholine were only $40-400 \mathrm{pA}$ when the cells were held at -50 $\mathrm{mV}$. The diameter of cultured sympathetic ganglion neurons ranges from 15 to $50 \mu \mathrm{m}$, and the single channel currents at $-50 \mathrm{mV}$ range from 2 to 4 pA (Moss et al., 1989), which leads to an estimated average receptor density of between 0.001 and 1 receptors $/ \mu \mathrm{m}^{2}$. Thus, a preganglionic growth cone that contacts a cultured sympathetic neuron is likely to encounter a surface virtually devoid of receptors. A corollary is that receptors must accumulate at developing synaptic sites before functional transmission can begin. Given the high input resistance of sympathetic ganglion neurons 
(often $>500 \mathrm{M} \Omega$ ), the first functional responses might be detectable when only a few receptors had accumulated at a postsynaptic site. However, as additional receptors accumulated at a synaptic site (and the mix of receptors shifted to larger conductance channels), the same quantity of acetylcholine would be expected to produce progressively larger amounts of depolarization. If synaptic sites develop asynchronously (as seems likely because there are multiple boutons), then substantial variation in quantal size between sites would be expected.

The receptor accumulation hypothesis also predicts that there should be some delay between the initial contact of cells and the first signs of functional transmission. Our ability to observe functional synapses at $48 \mathrm{~h}$ of co-culture but not at $24 \mathrm{~h}$ of co-culture may be a consequence of this process of receptor accumulation. Preganglionic neurons extend neurites rapidly under our culture conditions (Honig and Hume, 1986; Moorman and Hume, 1990). Based on the density of cells in the culture and the elongation rate of preganglionic neurites, the first physical contacts between preganglionic axons and ganglion cells seem likely to occur within $12-24 \mathrm{~h}$. Thus, in contrast to the situation at the neuromuscular junction, there may be a significant delay between initial contact and first function. Part of the explanation for the difference may be that uninnervated muscle cells express relatively high levels of acetylcholine receptors (Sytkowski et al., 1973), while uninnervated sympathetic ganglion neurons have only a very low level of responsiveness to acetylcholine (Role, 1988). It should be noted that the delay until the onset of functional transmission suggested by our studies of neurons in dissociated cell culture is longer than suggested by the report of Role (1988). She chowed that explants of spinal cord could produce excitatory responses in ganglion cells within $24 \mathrm{~h}$ of coculture. A possible explanation of the difference between the two studies is that the medium used to grow the cells differed substantially. The medium used in Role's experiments contained embryo extract and a much higher concentration of horse serum than was present in our experiments. Role showed that unidentified factors in the culture medium can significantly increase acetylcholine responsiveness, so it might be expected that the less rich medium we used would produce lower acetylcholine responsiveness. The very low initial responsiveness of the cells in our cultures to acetylcholine provides a plausible explanation of why we found a longer latency to initial synaptic function than did Role. It seems likely that the lower the initial re- sponsiveness to acetylcholine, the longer it would take to accumulate enough receptors to produce a detectable response. At present, the idea that there is a delay in the onset of synaptic function, due to a need to accumulate acetylcholine receptors, is only an inference. It will be interesting to directly test this prediction by following individual neurons over time.

We thank Drs. Beverly Clendening, Stephen Moorman, Steven Thomas, and James Walker for helpful comments on the manuscript and Ms. Nancy Hall and Mr. Mark Zawisa for excellent technical assistance. This research was supported by NIH grant NS2 1043 to R.H., who was a fellow of the Sloan Foundation during part of this work.

\section{REFERENCES}

Bekkers, J. M., Richerson, G. B., and Stevens, C. F. (1990). Origin of variability in quantal size in cultured hippocampal neurons and hippocampal slices. Proc. Natl. Acad. Sci. USA 87: 5359-5362.

Bevan, S. and SteinbaCH, J. H. (1977). The distribution of alpha-bungarotoxin binding sites on mammalian skeletal muscle developing in vivo. J. Physiol. (Lond.) 267:195-213.

Blackman, J. G., GinsborG, B. L., and Ray, C. (1963a) Spontaneous synaptic activity in sympathetic ganglion cells of the frog. $J$. Physiol. (Lond.) 167:389401.

Blackman, J. G., Ginsborg, B. L., and Ray, C. (1963b) On the quantal release of the transmitter at a sympathetic synapse. I. Physiol. (Lond.) 167:402415.

BoRnSTEIN, J. C. (1974). Multiquantal release of acetylcholine in mammalian ganglia. Nature 248:529-531.

BornsteIN, J. C. ( 1978). Spontaneous multiquantal release at synapses in guinea-pig hypogastric ganglia: evidence that release can occur in bursts. $J$. Physiol. (Lond.) 282:375-398.

BoYD, I. A. and MARTIN, A. R. (1956). The end-plate potential in mammalian muscle. $J$. Physiol. (Lond.) 132:74-91.

Clendening, B. and Hume, R. I. (1990). Expression of multiple neurotransmitter receptors by sympathetic preganglionic neurons in vitro. $J$. Neurosci. 10:39773991.

DEl CASTILlo, J. and KATZ, B. (1954). Quantal components of the end-plate potential. I. Physiol. (Lond.) 124:560-573.

Dennis, M. J., Harris, A. J., and Kuffler, S. W. (1971). Synaptic transmission and its duplication by focally applied acetylcholine in parasympathetic neurons in the heart of the frog. Proc. R. Soc. Lond. B. 177:509-539.

DREYeR, S. E. and ChiapPinelli, V. A. (1987). Analysis of quantal content and quantal conductance in two 
populations of neurons in the avian ciliary ganglion. Neuroscience 20:905-910.

Evers, J., LASer, M., SUN, Y., XIE, Z., and Poo, M. M. (1989). Studies of nerve-muscle interactions in Xenopus cell culture: analysis of early synaptic currents. $J$. Neurosci. 9:1523-1539.

FATT, P. and Katz, B. (1952). Spontaneous subthreshold activity at motor nerve endings. J. Physiol. (Lond.) 117:109-128.

Fenwick, E., Marty, A., and Neher, E. (1982). Sodium and calcium channels in bovine chromaffin cells. J. Physiol. (Lond.) 331:599-635.

FranK, E. and Fischbach, G. D. (1979). Early events in neuromuscular junction formation in vitro: induction of acetylcholine receptor clusters in the postsynaptic membrane and morphology of newly formed nerve-muscle synapses. $J$. Cell Biol. 83:143-158.

Hanker, J. S., Yates, P. E., Metz, C. B., and RusTIONI, A. (1977). A new specific, sensitive and noncarcinogenic reagent for the demonstration of horseradish peroxidase. Histochem J. 9:789-792.

HoniG, M. G. and Hume, R. I. (1986). Fluorescent carbocyanine dyes allow living neurons of identified origin to be studied in long-term cultures. $J$. Cell Biol 103: $171-187$.

Hume, R. I. and Purves, D. (1983). Apportionment of the terminals from single preganglionic axons to target neurones in the rabbit ciliary ganglion. J. Physiol. (Lond.) 338:259-275.

Hume, R. I., Role, L. W., and Fischbach, G. D. (1983). ACh release from growth cones detected with patches of $\mathrm{ACh}$ receptor rich membrane. Nature 303:632-634.

JACK, J. J., REDMAN, S. J., and WonG, K. (1981). The components of synaptic potentials cvoked in cat spinal motoneurones by impulses in single group IA afferents. J. Physiol. (Iond.) 321:111-126.

KIDOKoro, Y. and YeH, E. (1982). Initial synaptic transmission at the growth cone in Xenopus nerve muscle cultures. Proc. Natl. Acad. Sci USA 79:67276731 .

LILEY, A. W. (1956). An investigation of spontaneous activity at the neuromuscular junction of the rat. $J$. Physiol. (Lond.) 132:650-666.

MARTIN, A. R. (1955). A further study of the statistical composition of the endplate potential. I. Physiol (Lond.) 130:114-122.

Martin, A. R. and Pilar, G. (1964). Quantal components of the synaptic potential in the ciliary ganglion of the chick. J. Physiol. (Lond.) 175:1-16.
MCLaChlan, E. M. (1975). An analysis of the release of acetylcholine from preganglionic nerve terminals. $J$. Physiol. (Lond.) 245:447-466.

Moorman, S. J. and Hume, R. I. (1990). Growth cones of chick sympathetic preganglionic neurons in vitro interact with other neurons in a cell-specific manner. J. Neurosci. 10:3158-3163.

Moss, B. L., Scheutze, S. M., and Role, L. W. (1989). Functional properties and developmental regulation of nicotinic acetylcholine receptors of embryonic chicken sympathetic neurons. Neuron 3:597-607.

Nelson, P. G., Marshall, K. C., Pun, R. Y., ChrisTIAN, C. N., SherifF, W. H., JR., MaCDONALD, R. L., and NEALE, E. A. (1983). Synaptic interactions between mammalian central neurons in cell culture. II Quantal analysis of EPSPs. J. Neurophysiol. 49:14421458.

O'lague, P. H., Obata, K., Claude, P., Furshran, E. J., and POTTER, D. D. (1974). Evidence for cholinergic synapses between dissociatcd rat sympathetic neurons in cell culture. Proc. Natl. Acad. Sci. USA 71:3602-3606.

Purves, D. and Lichtman, J. W. (1985). Principles of Neural Development, Sinnauer Associates, Sunderland MA, p. 433.

RAHAMIMOFF, R. and YAARI, Y. (1973). Delayed release of transmitter at the frog neuromuscular junction. J. Physiol. (Lond.) 228:241-257.

REDMAN, S. (1990). Quantal analysis of synaptic potentials in neurons of the central nervous system. Physiot. Rev. 70:165-198.

RoLE, L. W. (1988). Neural regulation of acetylcholine sensitivity in embryonic sympathetic neurons. Proc. Natl. Acad. Sci. USA 85:2825-2829.

Sun, Y. and PoO, M. M. (1987). Evoked release of acetylcholine from growing embryonic neuron. Proc. Natl. Acad. Sci. USA 84:2540-2544.

SytKowski, A. J., VOGEL, Z., and NirenberG, M. W. (1973). Development of acetylcholine receptors on cultured muscle. Proc. Natl. Acad. Sci. USA 70:270274.

WALKER, J. J . and HUME, R. I. (1990). An in vitro analysis of the onset of synaptic transmission between chick autonomic neurons. Soc. Neurosci. Absir. 16:456.

YIP, J. W. (1987). Target cues are not required for the guidance of sympathetic preganglionic axons. Brain Res. 429:155-159.

Young, S. H. and Poo, M. M. (1983). Spontaneous release of transmitter from growth cones of embryonic neurons. Nature 305:637-639. 